\title{
Obtención de tableros de partículas a partir de residuo de desmote de algodón y resina urea formaldehído
}

\section{Obtaining particleboards from cotton gin residue and urea-formaldehyde resin}

Presentación: 01/06/2021

Aprobación: 29/09/2021

\begin{abstract}
Agustina Trevisan
Centro de Investigación y Desarrollo para la Construcción y la Vivienda CECOVI - Facultad Regional Santa Fe, Universidad Tecnológica Nacional - Argentina

atrevisan@frsf.utn.edu.ar
\end{abstract}

\section{Luciano Gabriel Massons}

Centro de Investigación y Desarrollo para la Construcción y la Vivienda CECOVI - Facultad Regional Santa Fe, Universidad Tecnológica Nacional - Argentina

Imassons@frsf.utn.edu.ar

\section{María Fernanda Carrasco}

Centro de Investigación y Desarrollo para la Construcción y la Vivienda CECOVI - Facultad Regional Santa Fe, Universidad Tecnológica Nacional - Argentina

mcarrasc@frsf.utn.edu.ar

\section{Rubén Marcos Grether}

Centro de Investigación y Desarrollo para la Construcción y la Vivienda CECOVI - Facultad Regional Santa Fe, Universidad Tecnológica Nacional - Argentina

rmgrethe@frsf.utn.edu.ar

\section{Ariel Anselmo González}

Departamento Ingeniería Civil - Facultad Regional Santa Fe, Universidad Tecnológica Nacional - Argentina aagonzal@frsf.utn.edu.ar

\section{Resumen}

El objetivo de este estudio fue la evaluación de la factibilidad de producción de tableros de partículas para uso general utilizando residuos de desmote de algodón generados en Argentina y resina urea formaldehído. Se investigó la composición química y granulometría del residuo de desmote, así como propiedades mecánicas y físicas de los tableros de partículas obtenidos. Se evaluó la densidad y la resistencia a la flexión de los tableros de partículas 
producidos con niveles variables de resina urea formaldehído entre 8.3 y 19.3 \% en relación sólido/sólido. También se analizó el efecto de la incorporación de refuerzo yute sobre las propiedades mecánicas de estos tableros. Se obtuvieron tableros de partículas con densidades entre 530 y $700 \mathrm{~kg} / \mathrm{m}^{3}$ y resistencia a flexión variable entre 0.30 y $5.85 \mathrm{MPa}$, permitiendo alcanzar los mínimos niveles requeridos para tableros de baja densidad.

Palabras clave: residuo de algodón, resina urea-formaldehído, tableros de partículas, yute.

\begin{abstract}
The objective of this study was the evaluation of the feasibility of producing particleboard for general use using cotton gin waste generated in Argentina and urea formaldehyde resin. The chemical composition and size distribution of particles of the ginning residue as well as mechanical and physical properties of the particleboards obtained were investigated. Density and flexural strength of particleboards produced with varying levels of urea-formaldehyde resin between 8.3 and $19.3 \%$ (solid to solid ratio) were evaluated. The effect of incorporating jute reinforcement on the mechanical properties of these boards was also analysed. Particle boards with densities between 530 and $700 \mathrm{~kg} / \mathrm{m}^{3}$ and variable flexural strength between 0.30 and $5.85 \mathrm{MPa}$ were obtained, allowing the minimum levels required for low-density boards to be reached.
\end{abstract}

Keywords: cotton gin residue, urea-formaldehyde resin, particleboards, jute.

\title{
Introducción
}

La producción algodonera en el norte de la provincia de Santa Fe es una importante actividad productiva que, junto a Chaco, Formosa, Santiago del Estero y Corrientes, muestran una fuerte concentración geográfica. Esta producción, presenta algunas problemáticas que deben ser atendidas.

En campañas anteriores, se obtuvieron aproximadamente $1.000 .000 \mathrm{t}$ de algodón en bruto, dejando atrás $300.000 \mathrm{t}$ (aprox. $1.195 .000 \mathrm{~m}^{3}$ ) de residuos (> $30 \%$ ) constituidos por fibrilla, cárpelos, materias extrañas, etc., sin destino previsto. En las últimas décadas se ha generalizado el empleo de cosecha mecánica, situación que mejora notablemente la rentabilidad del cultivo, pero a medida que se difunde, produce mayor cantidad de residuo de desmote que debe ser dispuesto de manera efectiva, generando inconvenientes y costos extraordinarios al sector desmotador.

Estos residuos, que habitualmente se acopian a cielo abierto resultan un hábitat de características excepcionales para alimañas y roedores y, asimismo, son autoinflamables por lo cual representan un peligro para las comunidades cercanas.

Otra característica del sector desmotador que resulta importante tener en cuenta es que realiza actividades intensivas durante aproximadamente 100 días al año, en correspondencia con las campañas de cosecha-desmote, dedicándose luego a actividades de mantenimiento u otras actividades conexas (procesamiento de semillas de algodón) con mínimo requerimiento de personal. Esta situación redunda en reducción de personal o reducción en la dedicación (y por lo tanto remuneración) de la población local.

Un aspecto de la producción algodonera que atenta contra la implementación de tecnologías de alta complejidad para el aprovechamiento de los residuos es la variabilidad 
de la producción interanual, registrándose en las últimas décadas campañas que oscilan entre 386.676 y 1.032 .545 t de algodón en bruto, de acuerdo a los datos del Ministerio de Agroindustria y de CCIA (Ministerio de Agricultura, 2016).

Actualmente se intenta utilizar los residuos de desmote como alimento balanceado para ganado, aunque se ve muy limitado por la baja digestibilidad del material, que supera apenas el $20 \%$, valor muy reducido en comparación con otras opciones de alimentos (Myer y Hersom, 2017; Young et al, 1979).

Otro uso está constituido por el compostaje, que aparenta ser la solución más viable, aunque su difusión como materia para elaborar compost es un tanto acotada y de baja rentabilidad (Ayers, 1997; Gordon et al, 2006).

Lamentablemente, en la mayoría de los casos se opta por calcinar estos residuos de desmote. Dado que la mayoría de estas plantas se encuentran dentro del radio urbano de ciudades, originan un serio problema de polución al cual no se le plantean soluciones y que provocan malestar entre los vecinos de los barrios aledaños, quienes temen sufrir algún tipo de afección respiratoria. En este sentido se deben considerar los riesgos asociados a la quema de residuos que podrían estar contaminados con agroquímicos (Crazov et al, 2001; 2003; 2000; Crossan et al, 2006).

Finalmente, si bien se identifican otros posibles usos como la hidrólisis o pirolisis, el alto valor agregado de los productos obtenibles se ve contrarrestado por el elevado costo de las instalaciones y de operación y la exigencia de mano de obra calificada.

En este escenario, la posibilidad de desarrollar elementos constructivos innovadores mediante la utilización de residuos del desmote del algodón podría ayudar a atender problemáticas ambientales y sociales de este sector agroindustrial, así como ofrecer nuevos materiales para su aplicación a la construcción o la fabricación de mobiliario sencillo. En este sentido, se vislumbran posibles aplicaciones a fin de mejorar las condiciones de habitabilidad de viviendas, ya que en los departamentos del norte de la provincia de Santa Fe se presentan porcentajes elevados de construcciones con serios problemas en sus envolventes verticales y horizontales (INDEC, 2010).

La elaboración de paneles basados en residuos lignocelulósicos constituye una tecnología de construcción en seco con características aislantes termoacústicas y buena resistencia a la degradación. Se han detectado numerosos trabajos que proponen la valorización de residuos mediante su empleo en aglomerados, algunos de ellos proponiendo estas tecnologías como sustitutos de paneles aglomerados de partículas de madera (Mendez y Sotelo, 2006; Contreras et al, 1999; Gatani et al, 2013; Medina y Ambrogi, 1994; Granero et al, 2013; Güller y Ozen, 2004; Varanda et al, 2013; Barbirato et al, 2014; Desirello et al, 2004; Pirayesh et al, 2013; Barros et al, 2011; Panyakaew, 2011; Xianjun et al, 2010). Especial interés despiertan algunas investigaciones desarrolladas en nuestro país, destinadas a la producción de paneles aglomerados a partir del marlo del maíz (Mendez y Sotelo, 2006), empleados como placas de cielorrasos livianos, aislantes y fáciles de instalar. Este tipo de elemento podría constituirse en una barrera mecánica para prevenir el ataque por la vinchuca y de este modo disminuir la aparición de Mal de Chagas.

Por su parte, la Dra. Mariana Gatani ha llevado adelante investigaciones en las que emplea cáscaras de maní para el desarrollo de paneles aglomerados destinados tanto a cerramientos y cielorrasos como a mobiliario de viviendas (Gatani et al, 2013; Granero et al, 2013).

En el presente trabajo se presentan algunos avances obtenidos en el proyecto con relación a las características del residuo, su acondicionamiento, la posibilidad de obtención de aglomerados, las propiedades alcanzadas y las perspectivas futuras identificadas para la investigación. 


\section{Materiales y métodos}

El residuo de desmote utilizado para la elaboración de los aglomerados se obtuvo de los acopios de una planta desmotadora ubicada en la provincia de Santa Fe (Argentina). Este residuo está constituido por restos de fibra de algodón que no se logra separar en el proceso industrial, carpelos, ramas de diversos tamaños, hojas y polvo que se incorpora durante el acopio (figura 1).

Estos residuos fueron triturados en un molino de martillos (LOYTO $\mathrm{N}^{\circ} 2$ ), provisto de una zaranda de $16 \mathrm{~mm}, 8$ martillos flotantes de acero y eje montado sobre bolilleros. Se efectuó la caracterización granulométrica del residuo de desmote molido y sin moler, previa homogeneización manual de las muestras y reducción del tamaño de estas por cuarteo. Para el análisis granulométrico se utilizaron tamices $\mathrm{N}^{\circ} 1 / 2$ ” $(12.5 \mathrm{~mm}), \mathrm{N}^{\circ} 3 / 8^{\prime \prime}(9.5 \mathrm{~mm}), \mathrm{N}^{\circ} 4$ (4.75mm), $\mathrm{N}^{\circ} 8(2.36 \mathrm{~mm}), \mathrm{N}^{\circ} 16(1.18 \mathrm{~mm})$ y $\mathrm{N}^{\circ} 30(0.6 \mathrm{~mm})$ sucesivamente, determinando el peso del material retenido en cada uno de ellos.

Sobre los residuos de desmote se realizaron determinaciones de $\mathrm{pH}$ (Sithole, 2005), solubilidad en $\mathrm{NaOH}$ al $1 \%$ (TAPPI 212 om-02), solubilidad en agua fría y caliente (TAPPI 207 om-93). Los valores correspondientes a solubilidad en agua fría y caliente, se corrigieron de acuerdo a su contenido de cenizas presente. Todas las determinaciones se realizaron por duplicado y los resultados que se presentan corresponden a las medias aritméticas de las réplicas.

Luego del proceso de molienda, el residuo se secó en estufa a $105 \pm 2{ }^{\circ} \mathrm{C}$ para reducir su contenido de humedad natural de aproximadamente $16 \%$ hasta un $3 \%$.

Posteriormente se realizó el mezclado manual del residuo de desmote con la resina urea formaldehido (Coladur 65\%) provista por Jucarbe S.A. (Argentina), con contenido de sólidos mínimo de $65 \%$, densidad de $1.26 \mathrm{~g} / \mathrm{cm}^{3}$, tiempo de gelación a $100{ }^{\circ} \mathrm{C}$ de 6 min y viscosidad a $25{ }^{\circ} \mathrm{C}$ de $950 \mathrm{cps}$. Se adicionó agua a la resina hasta alcanzar un contenido de sólido de $55 \%$ y se empleó como catalizador un $5 \%$ de solución saturada sulfato de amonio.

La proporción de resina utilizada para los aglomerados, en proporción al peso de residuo de desmote varió de 8.3 \% a 19.3 \%, estableciéndose como sólidos presentes en la resina en relación al peso del residuo.

Luego de homogeneizar la mezcla, se colocó en un molde, se prensó hasta alcanzar una presión máxima de $4.75 \mathrm{MPa}$ para luego mantener el material a $70^{\circ} \mathrm{C}$ de temperatura por 30 minutos, obteniendo tableros de dimensiones nominales de 170 x 170 x $10 \mathrm{~mm}$.

Se evaluó la utilización de tejido de yute bidireccional de densidad superficial $252 \mathrm{~g} / \mathrm{m}^{2}$, como refuerzo (figura 2). El mismo fue impregnado con la misma proporción de resina que el residuo de desmote (Desirello et al, 2004).

Sobre los aglomerados obtenidos se realizaron determinaciones de densidad y de resistencia a flexión.

La densidad se determinó de acuerdo al procedimiento de la norma IRAM 9705.

La determinación de la resistencia a flexión se realizó de acuerdo a los lineamientos de la norma IRAM 9706, manteniendo una longitud de probetas de $160 \mathrm{~mm}$. Para ello se cortaron las placas moldeadas en 4 probetas de dimensiones nominales $160 \times 40 \times 10 \mathrm{~mm}$. Estas probetas se acondicionaron durante 48 a 72 hs previas al ensayo, en un ambiente controlado a $20 \pm 2{ }^{\circ} \mathrm{C}$ y $65 \pm 5 \%$ de humedad relativa. La carga se aplicó en el centro de la luz entre apoyos, que se mantuvo igual a $140 \mathrm{~mm}$, con una velocidad de deformación constante de 12 $\mathrm{mm} / \mathrm{min}$, hasta que se verificó la falla de la probeta. 


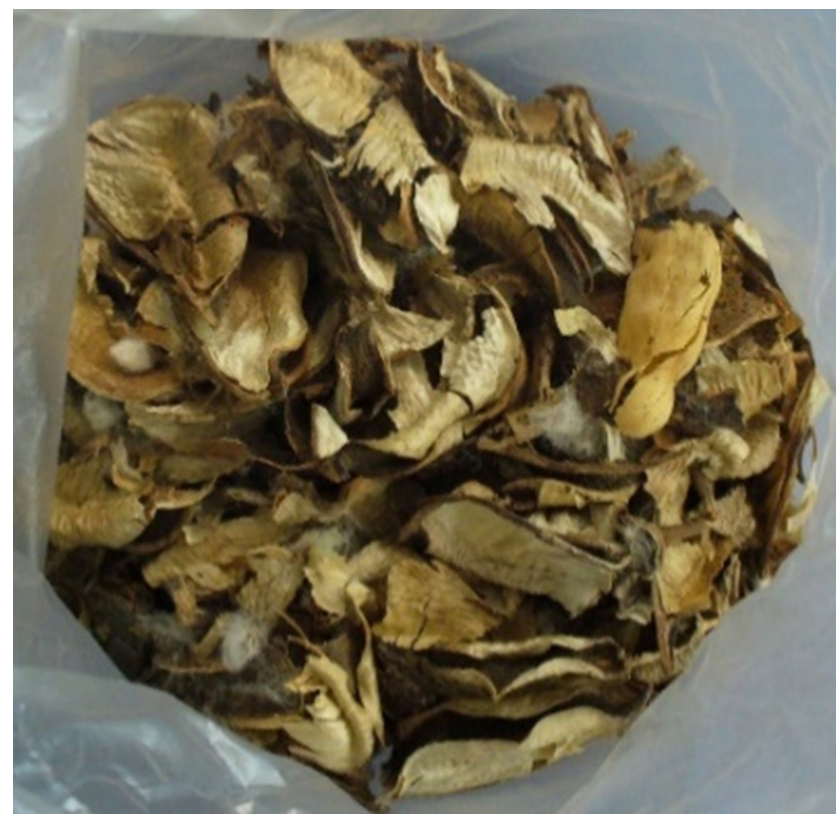

Figura 1: Residuo de desmote natural

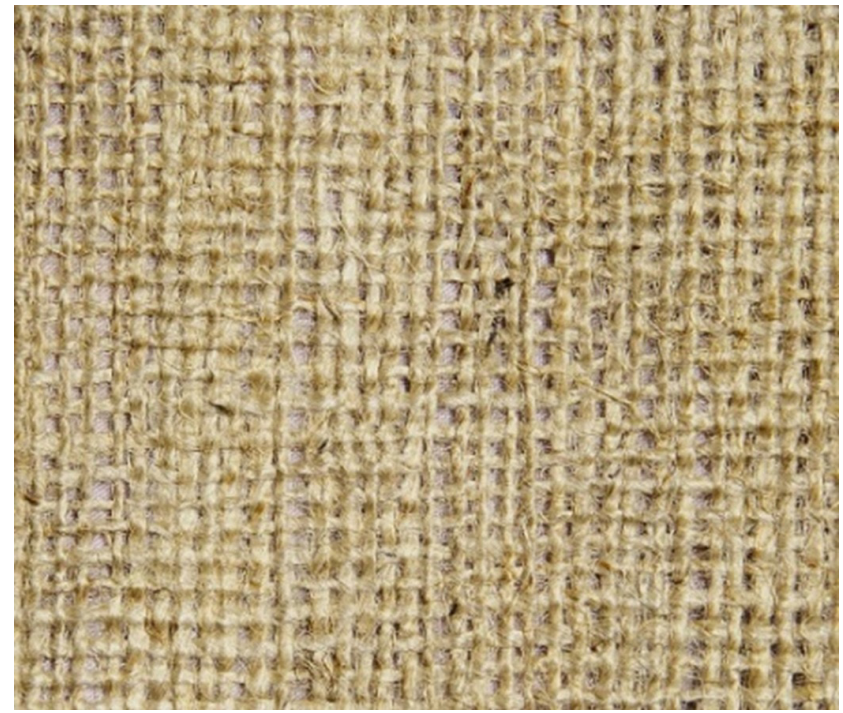

Figura 2: Tela de yute bidireccional

\section{Resultados y discusión}

El residuo de desmote posee formas cóncavas en su estado natural (figura 1), lo cual imposibilita una adecuada adherencia entre las partículas. Por este motivo se optó por realizar la molienda, logrando una mayor uniformidad en el tamaño de partículas y mejorando sus características morfológicas. 
En la Tabla 1 se pueden observar los porcentajes de residuo retenidos en cada tamiz, tanto para el residuo en su condición natural como para el residuo molido. Se advierte que la fracción mayor a $4.75 \mathrm{~mm}$ se reduce drásticamente mediante la molienda correspondiendo a un $95.85 \%$ en el residuo natural mientras que en el residuo molido alcanza el $54.8 \%$.

\begin{tabular}{|l|c|c|c|}
\hline \multicolumn{2}{|c|}{ Tamiz } & \multicolumn{2}{c|}{ Retenido (\%) } \\
\hline Nro. & Abertura (mm) & Residuo natural & Residuo molido \\
\hline $1 / 2^{\prime \prime}$ & 12.50 & $\cdots$ & 24.28 \\
\hline $3 / 8^{\prime \prime}$ & 9.50 & $\cdots$ & 8.86 \\
\hline 4 & 4.75 & 95.85 & 21.66 \\
\hline 8 & 2.36 & $-\ldots-1.05$ \\
\hline 16 & 1.18 & 1.93 & 13.16 \\
\hline 30 & 0.60 & $-\cdots$ & 6.13 \\
\hline 100 & 0.15 & 1.04 & $-\ldots$ \\
\hline Fondo & & 1.18 & 8.86 \\
\hline
\end{tabular}

Tabla 1: Granulometría del residuo de desmote

En el residuo molido se puede observar que presenta un porcentaje de $8.86 \%$ de partículas menores a $600 \mu \mathrm{m}$ que están constituidas por restos muy finos de partículas y polvo, que deben ser descartados para la fabricación de los aglomerados, debido a que resulta prácticamente imposible incorporarlos en forma homogénea mediante el mezclado.

Se aprecia que existe en el material molido una diversidad de tamaños de partículas entre 12.6 y $0.6 \mathrm{~mm}$, ya que se presentan porcentajes apreciables retenidos en cada uno de los tamices empleados. En este sentido, a partir de las fotografías de cada fracción retenida en los tamices (figura 3), pueden observarse diferentes morfologías de partículas, correspondiendo las más irregulares a los tamaños mayores y las más planas a los tamaños más pequeños.

Los resultados de solubilidad en $\mathrm{NaOH}$ al $1 \%$, en agua fría y en agua caliente y de $\mathrm{pH}$ del residuo se exponen en la tabla 2. El procedimiento de agua fría constituye una medida de componentes tales como taninos, gomas, azúcares y materiales colorantes. El procedimiento en agua caliente mide, además, el almidón, en tanto que las extracciones en $\mathrm{NaOH}$ evidencian la presencia de carbohidratos de bajo peso molecular principalmente las hemicelulosas presentes en la muestra.

La comparación de los valores de solubles presentes en el residuo de desmote, respecto de otros materiales tales como las maderas duras y blandas, rastrojos de cereales, y cáscaras provenientes de otros cultivos, como cáscara de maní y de avellanas, indica que estos valores son muy superiores a la mayoría de las especies utilizadas en otras investigaciones, con la sola excepción de las cáscaras de avellana (Cöpür, 2007; Eroglu, 1992; Güller et al, 2008). 


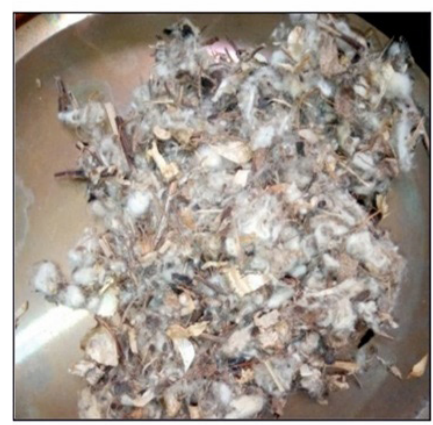

a)

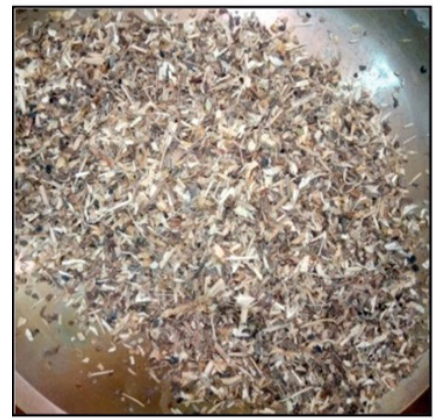

d)

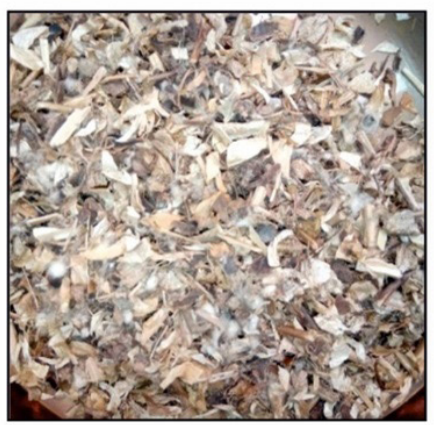

b)

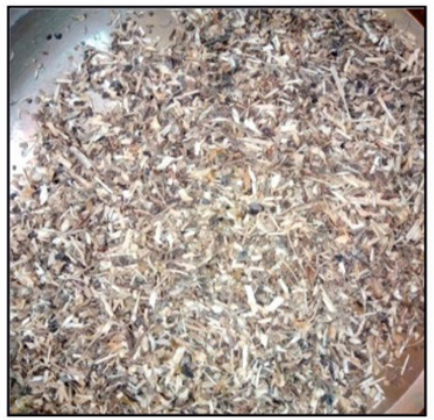

e)

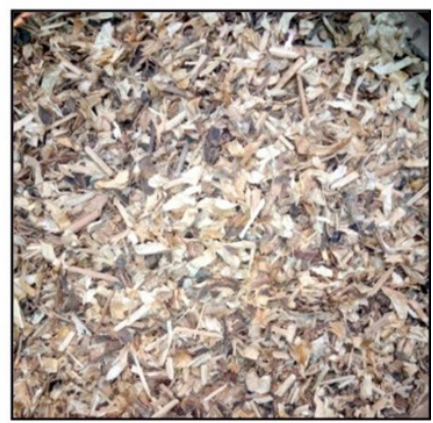

c)

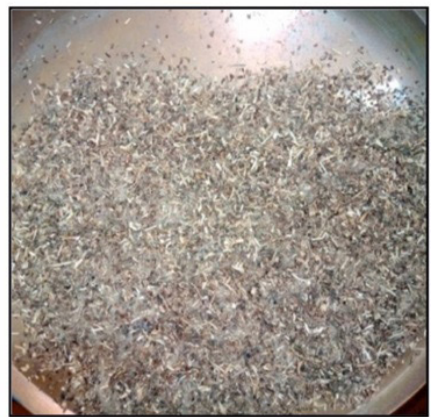

f)

Figura 3: Apariencia de las distintas partículas de residuo retenidas en cada tamiz: a) Retenido en $1 / 2$ "; b) Pasa $1 / 2$ ", ret. 3/8"; c) Pasa 3/8", ret. $4.75 \mathrm{~mm}$; d) Pasa $4.75 \mathrm{~mm}$, ret. $2.36 \mathrm{~mm}$; e) Pasa $2.36 \mathrm{~mm}$, ret. $1.18 \mathrm{~mm}$; f) Pasa $1.18 \mathrm{~mm}$, ret. $0.6 \mathrm{~mm}$.

\begin{tabular}{|l|c|}
\hline ANALISIS & VALOR \\
\hline Solubilidad en 1\% NaOH & $46.0 \%$ \\
\hline Solubilidad agua caliente & $16.1 \%$ \\
\hline Solubilidad agua fría & $11.9 \%$ \\
\hline pH agua fría & 5.0 \\
\hline pH agua caliente & 6.4 \\
\hline
\end{tabular}

Tabla 2: Determinaciones químicas realizadas sobre el residuo.

En la tabla 3 se observan los valores promedio de densidad y resistencia a flexión (MOR) obtenidos para los aglomerados elaborados con residuo de desmote. Las muestras producidas $\sin$ refuerzo se denominan $\mathrm{N}$ y aquellas en las que se incorpora una lámina de yute bidireccional en cada cara de identifican como N+Y. En la figura 4 se pueden observar imágenes descriptivas de estos paneles. 


\begin{tabular}{|c|c|c|c|c|c|c|}
\hline \multirow{2}{*}{ Identificación } & \multirow{2}{*}{$\begin{array}{c}\text { Presión } \\
(\mathrm{MPa})\end{array}$} & \multirow{2}{*}{ Resina } & \multicolumn{2}{|c|}{ MOR (MPa) } & \multicolumn{2}{c|}{ Densidad (kg/m $\left.\mathrm{m}^{3}\right)$} \\
\cline { 4 - 7 } & & & $\mathrm{N}$ & $\mathrm{N}+\mathrm{Y}$ & $\mathrm{N}$ & $\mathrm{N}+\mathrm{Y}$ \\
\hline $\mathrm{V}$ & 4.75 & $8.3 \%$ & 0.30 & 1.04 & 530 & 600 \\
\hline $\mathrm{U}$ & 4.75 & $11.9 \%$ & 1.62 & 3.06 & 610 & 600 \\
\hline $\mathrm{R}$ & 4.75 & $15.1 \%$ & 3.15 & 5.53 & 630 & 700 \\
\hline S & 4.75 & $19.3 \%$ & 3.05 & 5.85 & 630 & 670 \\
\hline
\end{tabular}

Tabla 3: Propiedades físicas y mecánicas de los aglomerados
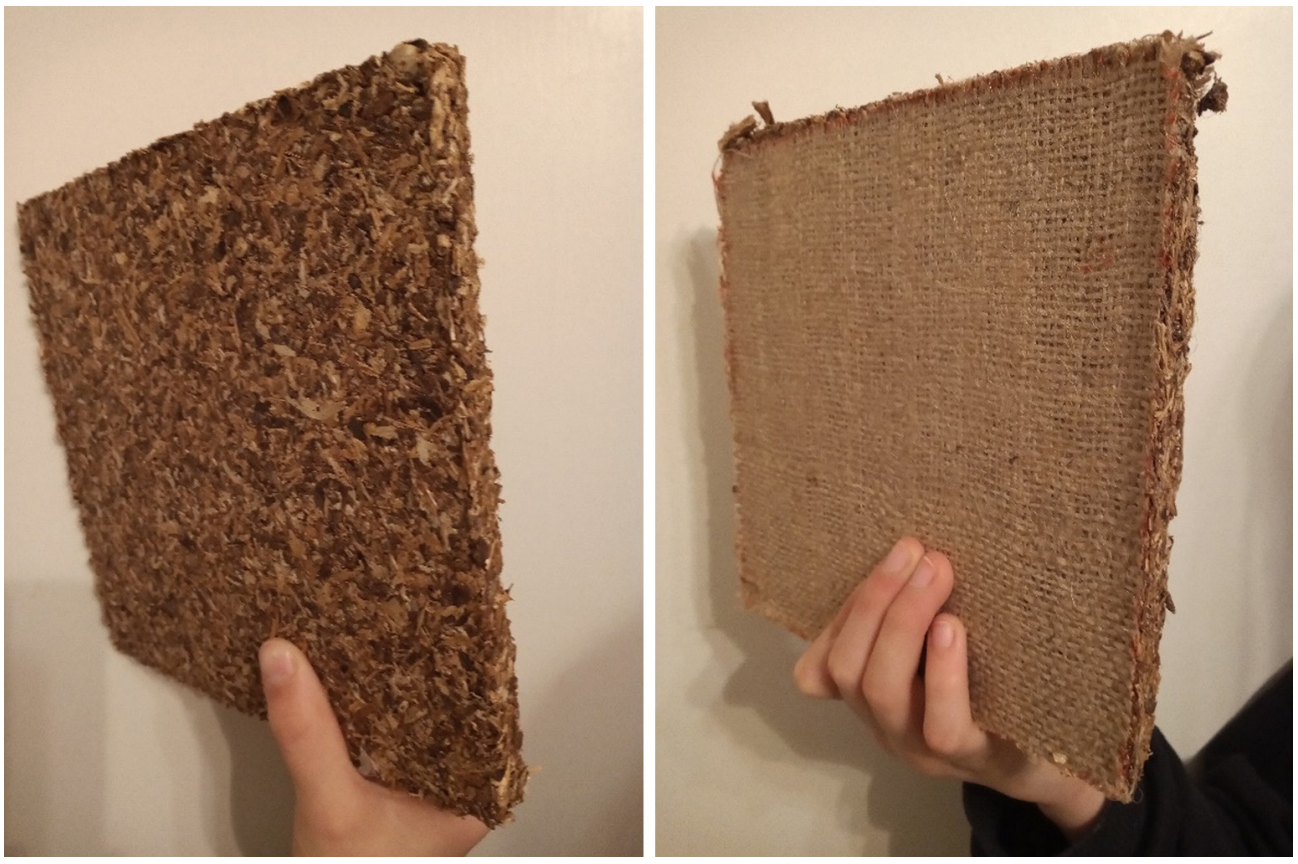

Figura 4: Paneles obtenidos en los moldeos: a) sin incorporación de refuerzo;

b) con incorporación de lámina de yute bidireccional.

La densidad de los aglomerados varía entre $0.53 \mathrm{~g} / \mathrm{cm}^{3}$ a $0.63 \mathrm{~g} / \mathrm{cm}^{3}$ para aquellos que no incorporan refuerzo $(\mathrm{N})$ y entre $0.60 \mathrm{~g} / \mathrm{cm}^{3}$ a $0.70 \mathrm{~g} / \mathrm{cm}^{3}$ para $\mathrm{los} \mathrm{N}+\mathrm{Y}$. En la siguiente figura se puede observar que a medida que se incrementa el contenido de resina, se produce un crecimiento de la densidad. Este comportamiento es similar para todos los aglomerados, independientemente de la presencia de refuerzo (Fig. 5), hasta un contenido de $15.1 \%$.

Los valores de densidad obtenidos colocan a los aglomerados sin refuerzo en la clasificación de baja densidad (LD) según la norma ANSI A208.1 (ANSI, 1999), que establece un límite superior de $640 \mathrm{~kg} / \mathrm{m}^{3}$. Paralelamente, la incorporación del refuerzo de yute bidireccional incrementa los valores de densidad, alcanzando los aglomerados con mayor porcentaje de resina la clasificación de media densidad (M) según ANSI A208.1 (640a 800 kg/m³) (ANSI, 1999). 
Los resultados de resistencia a flexión (MOR) de los aglomerados producidos (tabla 3) indican que el aumento en el contenido de resina permite incrementar los valores de resistencia a flexión, tanto en los casos de aglomerados simples como en aquellos en los que se incorporan refuerzos. La norma ANSI A208.1 (ANSI, 1999) establece un valor mínimo de resistencia a flexión de $3 \mathrm{~N} / \mathrm{mm}^{2}$ para los paneles LD-1 y de $5 \mathrm{~N} / \mathrm{mm}^{2}$ para los clasificados como LD-2. En el caso de los paneles de media densidad (M) el valor exigido de resistencia a flexión corresponde a $11 \mathrm{~N} / \mathrm{mm}^{2}$.

Los resultados indican que, el incremento en la densidad del material y del contenido de resina, tienen un efecto positivo en el desarrollo de resistencia (Fig. 6). En la figura 7 se puede observar que a medida que se incrementa el contenido de resina, crece la diferencia de resistencia entre los tableros con y sin yute, esto puede explicarse dado que la adherencia entre el yute y el residuo de desmote de algodón también aumentan.

$\mathrm{Al}$ colocar el yute, si bien la densidad no cambia significativamente, aumenta la resistencia del aglomerado, ya que el yute actúa como refuerzo mecánico, pudiéndose obtener un aumento en la resistencia que ronda los $3 \mathrm{MPa}$ para $19.3 \%$ de resina, lo cual permitió alcanzar valores establecidos por la norma ANSI A208.1 (ANSI, 1999). Este efecto resulta poco significativo para los menores contenidos de resina, ya que hasta $11,90 \%$ de incorporación se aprecia que este refuerzo tiende a despegarse del aglomerado al acercarse la falla (figura 8a). Contrariamente, para contenidos de resina de 15,1 y 19,3\% el yute se mantiene adherido y se observa la rotura de sus fibras, mostrando una efectiva colaboración para la mejora del comportamiento mecánico.

De acuerdo a la norma ANSI A208.1 (ANSI,1999), los paneles clasificados como correspondientes a baja densidad (LD-1 y LD-2) se reservan para su aplicación en interiores de puertas, es decir, para usos sin exigencias de carácter estructural. De acuerdo a estos criterios, los tableros identificados como U, R y S (tanto con o sin refuerzo) podrían emplearse para esos fines y, en combinación con otros materiales que aporten mayor resistencia en la conformación de placas compuestas para cielorrasos. Así, se requerirá incrementar la resistencia a flexión de los tableros producidos, hasta un mínimo de $11 \mathrm{~N} / \mathrm{mm}^{2}$, para permitir su empleo en otras aplicaciones comerciales, industriales y de construcción.

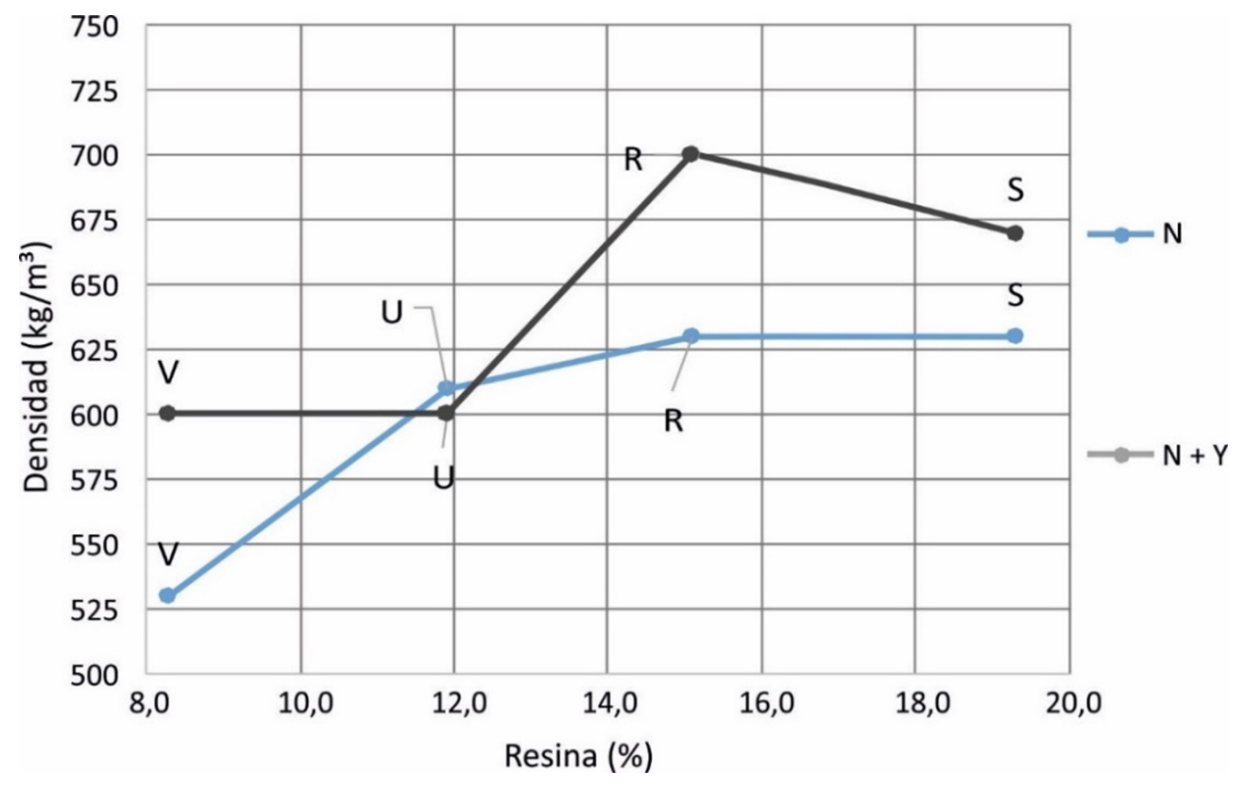

Figura 5: Densidad de los aglomerados 


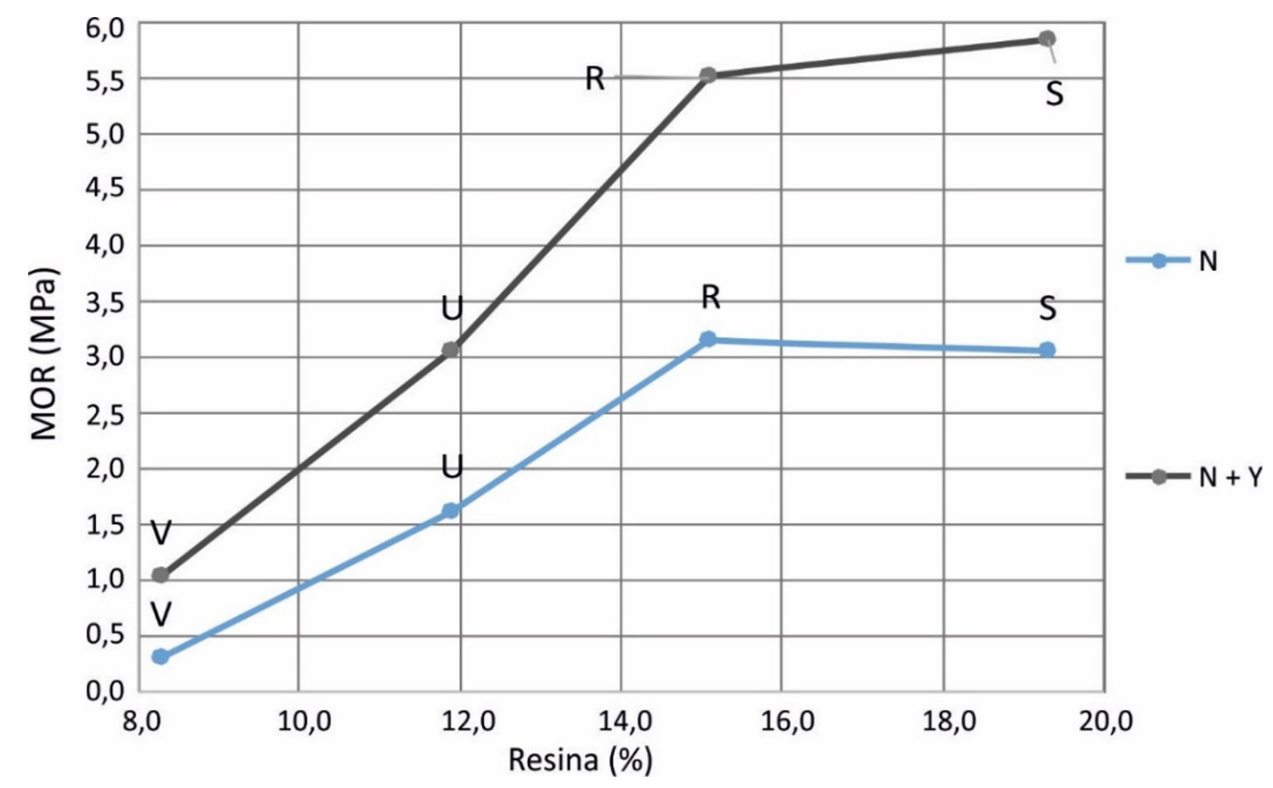

Figura 6: Resistencia a flexión de los aglomerados

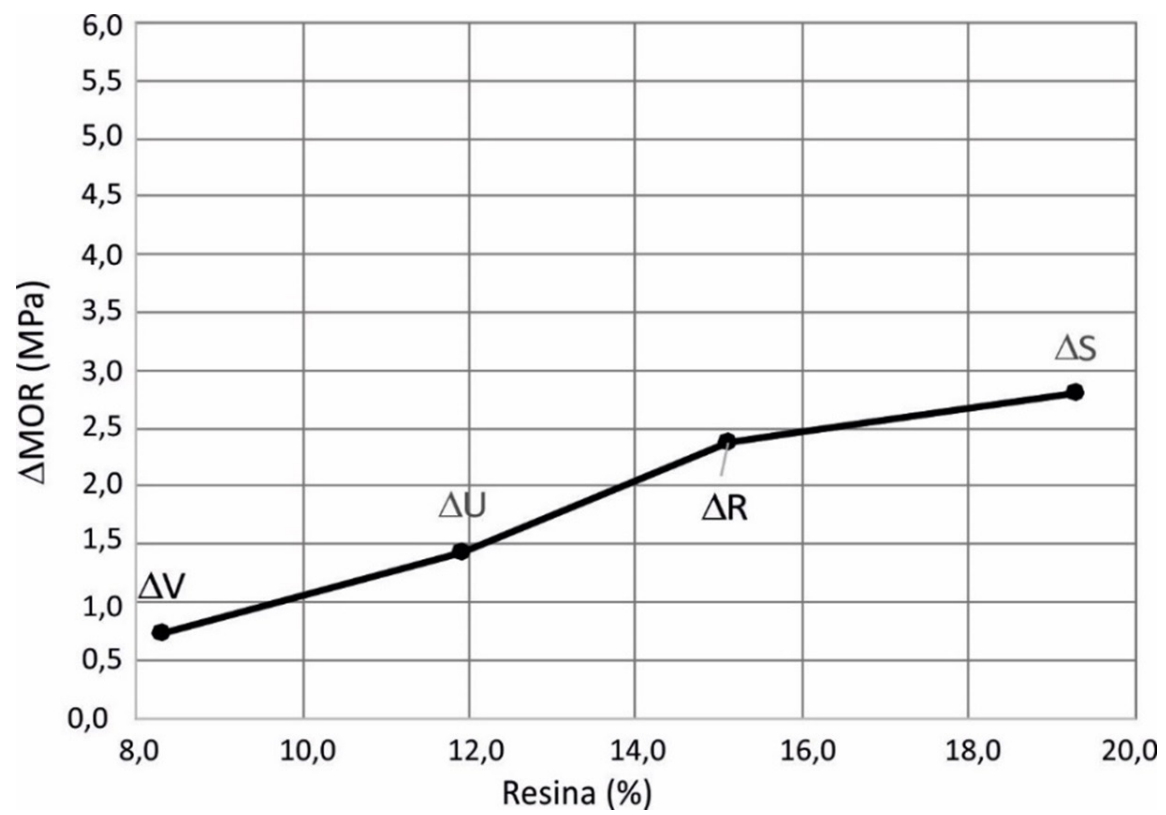

Figura 7: Incremento en la resistencia a flexión de los aglomerados debido a la incorporación de refuerzo

Si bien no se han detectado otras investigaciones que empleen el residuo de desmote completo, se han podido identificar trabajos de otros autores que utilizan los tallos de las 
plantas de algodón y los carpelos, respectivamente (Güller y Ozen, 2004; Khanjanzadeh et al, 2012; Güller, 2015). Güller utilizó tallos de las plantas de algodón para la elaboración de aglomerados con contenidos de resina urea formaldehído de 8\% para la capa central y $10 \%$ para las capas exteriores. Para estos aglomerados obtuvo densidades de 600 y $800 \mathrm{~kg} / \mathrm{m}^{3}$ y resistencia a flexión de 11.4 y $15.67 \mathrm{MPa}$, respectivamente (Güller, 2015). Este mismo autor obtuvo, para aglomerados de tallos de algodón y resina urea formaldehido, valores de resistencia variables en función del contenido de resina de las capas internas (ML) y externas (OL) y de la densidad final de $4.38 \mathrm{MPa}$ (densidad 400 $\left.\mathrm{kg} / \mathrm{m}^{3}-10 \% \mathrm{ML}-12 \% \mathrm{OL}\right), 8.79 \mathrm{MPa}$ (densidad $\left.500 \mathrm{~kg} / \mathrm{m}^{3}-10 \% \mathrm{ML}-12 \% \mathrm{OL}\right), 12.36$ $\mathrm{MPa}$ (densidad $600 \mathrm{~kg} / \mathrm{m}^{3}-10 \% \mathrm{ML}-12 \% \mathrm{OL}$ ) y $16.79 \mathrm{MPa}$ (densidad $700 \mathrm{~kg} / \mathrm{m}^{3}-10 \%$ ML - 12 \% OL) (Güller y Ozen, 2004 ). Hakki Alma et al. elaboraron aglomerados con los carpelos de la planta del algodón con contenidos de resina urea formaldehído de 9 $\%$ para la capa central y $11 \%$ para las capas exteriores. Para estos aglomerados obtuvo densidades de 668 a $693 \mathrm{~kg} / \mathrm{m}^{3}$ y resistencia a flexión de aproximadamente 10.5 y 11.5 MPa. (Hakki Alma et al; 2005).

Resulta evidente que la resistencia obtenida para los aglomerados elaborados con el residuo de desmote completo se ve afectada por la heterogeneidad del mismo y la presencia de partículas de formas y tamaños diferentes como lo son los carpelos, ramas de diversos tamaños y hojas, ya que resulta muy complejo el desarrollo de las uniones entre estos componentes. Así también se ve disminuida por la presencia de fibras de algodón, que, por su gran superficie y aglomeración, complican la impregnación completa y uniforme con resina, generándose núcleos de resina-fibras pobremente unidos en donde la resistencia disminuye considerablemente.

Paralelamente, es imprescindible tomar en consideración que la norma ANSI A208.1 (ANSI, 1999) establece un límite para las emisiones de formaldehido de $0.30 \mathrm{ppm}$, en tanto la norma IRAM 9723 establece valores límite de $8 \mathrm{mg} / 100 \mathrm{~g}$ para los tableros clase E1 y de entre 8 y $20 \mathrm{mg} / 100 \mathrm{~g}$ para los tableros clase E2. Hakki Alma et al. obtuvieron niveles de emisión de formaldehido entre 42,5 y 47,3 $\mathrm{mg} / 100 \mathrm{~g}$ para los tableros elaborados con carpelos de algodón con $11 \%$ de resina en las capas exteriores y $9 \%$ en la capa central (Hakki Alma et al; 2005). Por otra parte, Pirayesh et al. obtuvieron para los mismos contenidos de resina utilizada en paneles hechos con $100 \%$ de cáscara de nuez valores de emisión de formaldehído de $4,9 \mathrm{mg} / 100 \mathrm{~g}$. Estos resultados muestran que el incremento necesario para alcanzar niveles mínimos de resistencia en los tableros elaborados con el residuo de desmote completo podrían provocar emisiones muy superiores a los límites exigidos para proteger la salud, por lo cual deberán ser evaluados y, en caso de ser necesario, recurrir a medidas tales como la adición de secuestrantes, dado que se ha descubierto que la mezcla de tanino hidrolizado con resina UF reduce significativamente los niveles de emisión de formaldehído (Bisanda et. al., 2003). 


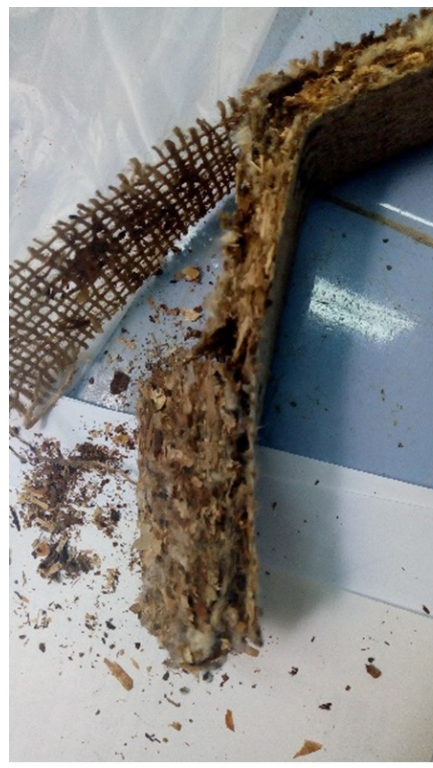

a)

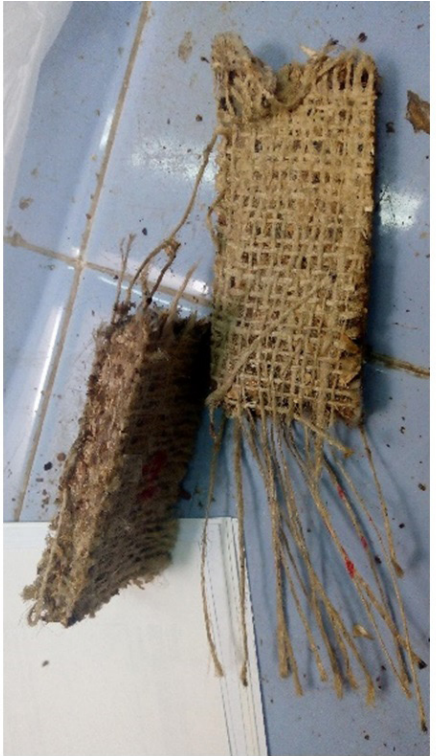

b)

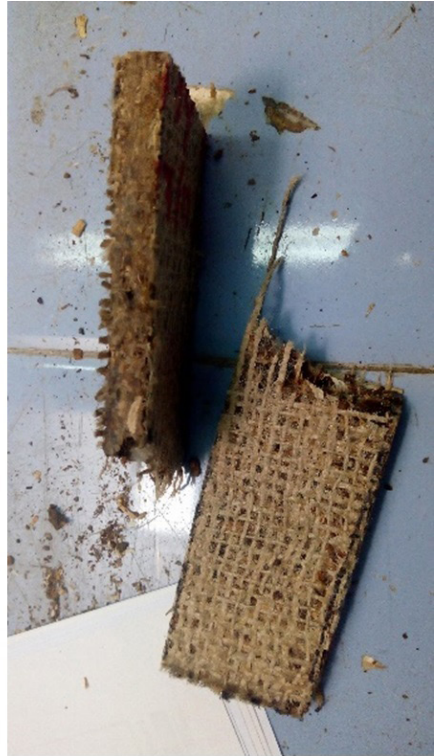

c)

Figura 8: Modo de falla de los aglomerados reforzados con yute: a) probetas $U(\mathrm{~N}+\mathrm{Y})$; b) probetas $\mathrm{R}(\mathrm{N}+\mathrm{Y})$; c) probetas $\mathrm{S}(\mathrm{N}+\mathrm{Y})$.

\section{Conclusiones}

A partir de los resultados obtenidos en la investigación, se puede concluir que:

- Es posible producir aglomerados a partir de los residuos del desmote y resina urea-formaldehido con propiedades cercanas a tableros comerciales de baja densidad, cumpliendo con los requisitos mínimos exigidos por las normas.

- Las características del residuo provocan importantes demandas de resina para lograr un buen comportamiento físico y mecánico del aglomerado, lo que se traduce en mayores costos de producción.

- La incorporación de refuerzos de yute incrementa significativamente la resistencia a flexión de los aglomerados, permitiendo alcanzar los niveles requeridos para tableros de baja densidad por la norma ANSI 208.1, sin incorporar pasos complejos para el proceso productivo.

- Los elevados contenidos de resina urea - formaldehido se relacionan con considerables emisiones de formaldehido, por lo que se evaluarán alternativas de reemplazo de esta resina por otra de mayor compatibilidad con el residuo o la incorporación de aditivos secuestrantes.

\section{Agradecimientos}

La concreción de este trabajo fue posible gracias a la colaboración de la desmotadora de algodón ACRIBA S.A de Villa Minetti (Santa Fe), al financiamiento de la Secretaría de Ciencia, Tecnología y Posgrado de la Universidad Tecnológica Nacional y a la colaboración del Instituto de Tecnología Celulósica de la FIQ-UNL. 


\section{Referencias}

ANSI 208.1. Particleboard. American National Standards Institute, 1999.

Ayers, V. "Farmer Composting of cotton gin trash". Reprinted from the Proceedings of the Beltwide Cotton Conference. Volume 2:1615-1616, pp 1615-1616. National Cotton Council, Memphis TN (EEUU), 1997.

Barbirato, G.; Fiorelli, J.; Barrero, N.G.; Agnolon Pallone, E.M.J.; Rocco Lahr, F. A.; Christoforo, A.L.; Savastano Jr. H. (2014). Painel aglomerado híbrido de casca de amendoim reforçado com partículas de madeira Itaúba. Ciência Florestal, 24(3), 685-697.

Barros Filho; R.M., Mendes, L.M., Monteiro Novack; K., Oliveira Aprelini, L., Botaro, V.R. (2011). Hybrid chipboard panels based on sugarcane bagasse, urea formaldehyde and melamine formaldehyde resin. Industrial Crops and Products, 33, 369-373.

Bisanda, E.T.N., Ogola, W.O.; Tesha, J.V. (2003) Characterisation of tannin resin blends for particle board applications. Cement \& Concrete Composites 25 593-598

Contreras M. W., Owen de C. M. E., Garay J. D. A., Contreras M. Y. (1999). Elaboración de tableros aglomerados de partículas de caña brava (Gyneriumsagittatum) y adhesivo urea-formaldehído. Rev. Forest. Venez. 43 (2), 129-135.

Cöpür, Y., Güller, C., Akgül, M., Tascioglu, C. (2007). Some chemical properties of hazelnut husk and its suitability for particleboard production. Building and Environment 42 2568-2572, 2007.

Cravzov, A. L., Chomiczak, S., Dalla Fontana, L., Marinich, J. "Evaluación del uso de plaguicidas en cultivos de algodón”. In: VI Reunión de Comunicaciones Científicas y Tecnológicas de la UNNE, Resistencia, Chaco (Argentina), 2000.

Cravzov, A. L., Traskauskas, C., Delfino, M. R. "Pesticidas en semillas de algodón y desecho de desmote (Pesticides in cotton seeds and ginning trash)". In: XII Congreso Argentino de Toxicología - XXI Jornadas Interdisciplinarias de Toxicología - I Jornada Rioplatense de Toxicología. Rosario, Santa Fe (Argentina), 2001.

Cravzov, A. L., Delfino, M. R., Avallone, C. M. Traskauskas, C., Montenegro, S. Tauguinas, A. "Carbamatos en semillas de algodón y desecho de desmote". In: IX Reunión de Comunicaciones Científicas y Tecnológicas de la UNNE, Resistencia, Chaco (Argentina), 2003.

Crossan, A., Sanchez Bayo, F., Kennedy, I., Bodnaruk, K. (2006). Risk of pesticide contamination in cotton seed and livestock. The Australian cottongrower, 27 (1), 54-56.

Desirello, C., Cerini, S., Charadia, R., Scalfi, R., Liberman, C., Stefani, P. M. Efecto de las condiciones de procesado sobre las propiedades mecánicas de aglomerados de cáscara de arroz. CONGRESO SAM/CONAMET 2004 . UNL. Santa Fe, Santa Fe (Argentina), 2004.

Eroglu H. (1992). Fiberboard industry. Karadeniz Technical University Publication 304, 212.

Gatani, M.P.; Fiorelli, J.; Medina, J.C.; Arguelo, R.; Ruiz, A.; Nascimento, M.F.; Savastano Jr., H. (2013). Viabilidade técnica de produção e propriedades de painéis de partículas de casca de amendoim. Revista Matéria, 18 (2), 1286-1293.

Gordon, E., Keisling, T. C., Oliver, L. R., Harris, C. (2006). Two methods of composting gin trash. Communications in Soil Science and Plant Analysis 32 (3-4), 491-507.

Granero, V.; Gatani, M.; Medina, J. C.; Ruiz, A.; Fiorelli, J., Kreiker, J., Lerda, M. J. (2013). Determinación de la influencia del tamaño y forma de partículas de cáscaras de maní en paneles aglomerados. Revista Quebracho Vol. 21(1,2),67-80. 
Güller, C.; Ozen, R. (2004). Some properties of particleboards made from cotton stalks (Gossypium hirsitum L.). HolzRohWerkst 62, 40-43.

Güller, C., Cöpür, Y., Tascioglu, C. (2008). The manufacture of particleboards using mixture of peanut hull (Arachis hypoqaea L.) and European Black pine (Pinus nigra Arnold) wood chips. Bioresource Technology 99, 2893-2897.

Güller, C. (2015). Research on the production of the composite panels from some agricultural residues. Pro Ligno vol. 11 (4), 187-191.

Hakki Alma, M., Kalaycioglu, H., Bektas, I., Tutus, A. (2005) Properties of cotton carpel-based particleboards. Industrial Crops and Products 22 141-149.

Instituto Nacional de Estadísticas y Censos. Censo Nacional de Población, Hogares y Vivienda 2010. Recuperado el 10 de Marzo de 2013 de http://www.censo2010.indec.gov.ar/ resultadosdefinitivos_totalpais.asp.

Khanjanzadeh, H., Bahmani, A.A., Rafighi, A, Tabarsa, T. (2012). Utilization of bio-waste cotton (Gossypium hirsutum L.) stalks and underutilized paulownia (paulownia fortunie) in wood-based composite particleboard”. African Journal of Biotechnology, 11(31), 8045-8050.

Medina, J.C., Ambrogi, A. (1994). Cáscara de maní en la elaboración de aglomerados, Revista Quebracho, Vol. 2, $47-53$.

Mendez, L.I. y Sotelo, R.M. Reciclo del Marlo de Maíz. Tableros de Partículas. Reciclado de residuos de construcción y demolición (RCD) y de residuos de procesos (RP)PROCQMA, ISBN 950-42-0056- 7, Universidad Tecnológica Nacional. San Rafael, Mendoza (Argentina), abril 2006.

Ministerio de Agricultura, Ganadería y Pesca de la Nacíon. Informe sobre situación actual y perspectivas de la producción algodonera Argentina. $69^{\circ}$ Reunión del CCIA (Comité Consultivo Internacional del Algodón); Lubbock, Texas (EEUU), 2016.

Myer. R. O., Hersom M. (12 de noviembre de 2017). Cotton Gin Trash: Alternative Roughage Feed for BeefCattle. Animal Science Department, Florida Cooperative Extension Service, Institute of Food and Agricultural Sciences, University of Florida. Recuperado el 24 de mayo de 2021 de http://edis. ifas.ufl.edu.

Panyakaew, S., Fotios, S. (2011). New thermal insulation boards made from coconut husk and bagasse. Energy and Buildings, 43, 1732-1739.

Pirayesh, H., Khanjanzadeh, H., Salari, A. (2013). Effect of using walnut/almond shells on the physical, mechanical properties and formaldehyde emission of particleboard. Composites: Part $B$ 45, 858-863.

Sithole, B. (2005). New method of measuring the $\mathrm{pH}$ of wood chips. Pulp and Paper, 106 (11), 42-45.

Varandaa, L.D., do Nascimento, M.F., Christoforo, A.L., Lopes Silva, D. A., Rocco Lahr, F.A. (2013). Oat Hulls as Addition to High Density Panels Production. MaterialsResearch, 16(6), 13551361.

Xianjun, L., Zhiyong, C., Winandy, J.E., Basta, A.H. (2010). Selected properties of particleboard panels manufactured from rice straws of different geometries. Bioresource Technology, 101, 4662-4666.

Young, K. B., Ahmed, M. U. (1979). Economics of uising cotton gin trash as a supplemental feed for range cattle. Journal of range management 32 (2), 123-126. 